\title{
„Der wahre Jacob“ und „Kikeriki“. Jüdische und proletarische Körper in satirischen Zeitschriften der \\ zwanziger Jahre
}

\author{
JULIA SCHÄFER
}

Der kriegsversehrte, defiziente Körper nach dem Ersten Weltkrieg weckte die Sehnsucht nach einer Gegen-Repräsentation, einem gesunden, intakten Körper. Präsent war der verletzte Mann mit amputierten Gliedern und entstelltem Gesicht sowohl im Straßenbild als auch in der bildenden Kunst (bei Otto Dix oder George Grosz) oder im massenmedialen Raum in Form der medizinischen Dokumentation in Fotografie und Film (vgl. Friedrich 1924/2004 sowie den Beitrag von Sabine Flach in diesem Band). Durch die Transzendierung des „Körper sein“ gelangte die bürgerliche Mittelschicht der Weimarer und der Ersten Republik zur Rationalisierung eines „Körper haben“, der Hoffnung auf einen zugerichteten und formbaren Körper im Rahmen der technischen Machbarkeit.

Die massenmediale Visualisierung von Körperkorrekturapparaten oder kriegs- und krisentypischen Erkrankungen wie Rachitis, Plattfüßen und kontagiösen Krankheiten wie Tuberkulose oder Syphilis macht deutlich, wie sich das medizinische Expertenwissen in die Populärkultur einschreibt. Dieser Prozess soll im Folgenden anhand der medikalisierten Bilder von jüdischen und proletarischen Körpern in satirischhumoristischen Zeitschriften der Weimarer bzw. Ersten Österreichischen Republik, dem sozialdemokratischen Der Wahre Jacob (1884-1933) und dem christlichsozialen Kikeriki (1867-1933), rekonstruiert werden. An- 
hand dieser Bilder lässt sich insbesondere dokumentieren, inwiefern Körpermerkmale als kulturelle Bedeutungsträger innerhalb eines Diskurses der Differenz fungieren (vgl. Schäfer 2005).

Diese populären Repräsentationen sind aber auch deshalb von Interesse, weil sie durch Referenz auf die zeitgenössischen Wissenschaften (wie Rassenanthropologie, Psychopathologie, Physiognomik bzw. Pathognomik) ihre Bedeutung über das einzelne Medium und seine spezielle Leserschaft hinaus generalisieren. Der jüdische und der proletarische Körper werden dabei zu propagandistischen Projektionsfeldern und ,Kollektivsymbolen“ (Drews/Link/Gerhardt 1984: 256-375; Becker/Gerhardt/Link 1997: 70-154), die verschiedene Ressentiments in tradierten Bildern bündeln.

\section{Zum Verhältnis von jüdischen und proletarischen Körperbildern}

Einige Merkmale des bürgerliche Körperideals lassen sich an Werbeannoncen, wie sie in den zwanziger Jahren in populären Zeitschriften publiziert wurden, ablesen: „Nasenformer“ (in Kombination mit einem „Stirnrunzelglätter“) oder „Beinkorrektionsapparate“ eröffneten individuelle Möglichkeiten zur Nachbesserung der physiognomischen oder orthopädischen Phänomene (Abb. 1). Aus gekrümmten, höckerigen Nasen und krummen Beinen sollten so gerade und ebenmäßige Formen werden, die nicht mehr auffielen. Als Prototyp fand sich die gerade,

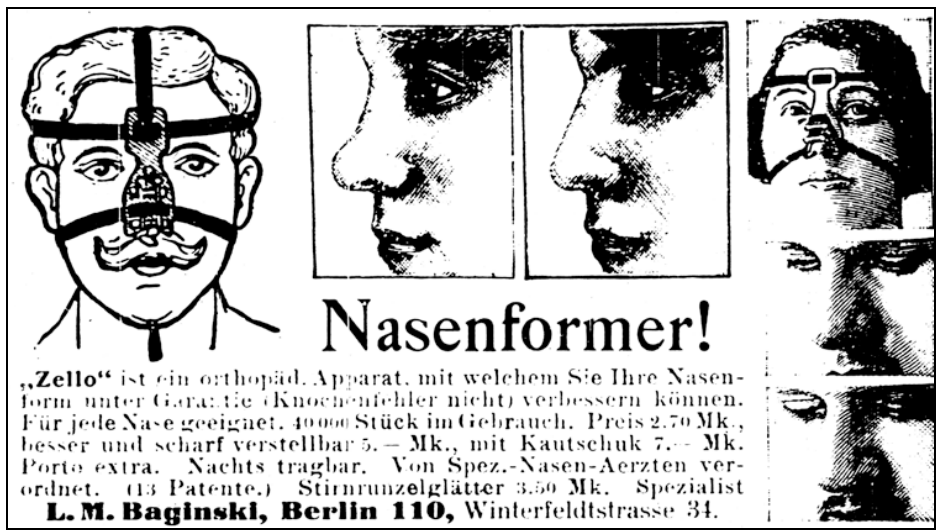

Abb. 1: Nasenformer. Werbeanzeige. 
deutsch/,arisch“ markierte Nase, als Kollektivsymbol und Wunschbild, im Kontrast zur bäuerlich oder proletarischen Himmelfahrts- bzw. Säufernase oder der jüdischen gebogenen, herabhängenden Hakennase (vgl. Schäfer 2004: 221f.). Eingerahmt waren diese Korrekturapparate meist von Annoncen für Fahrräder, Bruchbänder, „Körperbildungssysteme“ zur Entwicklung der Körpergröße um 10-15 cm, „Büstenverschönerer“ und Brustkamellen. Der Nasenformer, der nur geeignet sein sollte für die Korrektur der weichen, knorpeligen Bestandteile der Nase („Knochenfehler nicht!"), fand auch eine ironisch-sarkastische Kommentierung, etwa im jüdischen Witzblatt Schlemiel als „Nasenformer Antisemit“, der allen „Assimilanten und anderen Deutschnationalen“ (Schlemiel 4/1919: 94) angepriesen oder im Kikeriki „Renegaten empfohlen [wurde]. Wie man leicht entnationalisiert werden kann“ (Kikeriki 30/1929: 5).

Medizinhistorisch betrachtet, bilden diese Praktiken ein wichtiges frühes Kapitel der modernen kosmetischen Chirurgie, die O-/X-Beine oder Haken-/Knollennasen zu normalen, ebenmäßigen verformte. Dabei waren sich die Pioniere der Schönheitschirurgie, wie der Berliner Arzt Jacques Joseph, des assimilatorischen Impetus ihrer Patienten, die ihre ,semitische“ Nasenform gegen eine der Mehrheitsgesellschaft, die sie mit der deutschen identifizierten, eintauschen wollten, bereits bewusst (vgl. Berliner Klinische Wochenschrift 1898 ; Gilman 2000). Schönheit erscheint hier nicht mehr als Natur, sondern als Kunst.

Dieser Vorstellung von Machbarkeit widersprechen indes die scheinbar fixierten Körper von Juden und Proletariern in den oben angeführten satirischen Zeitschriften. Ich möchte den Versuch machen, die visuellen Stereotype von Arbeitern und Juden als Verkörperung von Rasse und Klasse daraufhin zu befragen, ob sie austauschbare Variablen der Diskriminierung sind. Ikonisch, also hinsichtlich der zuordenbaren Bildzeichen, die wiederum in körperliche Zeichen und sachliche Attribute unterschieden werden, sind diese Motive zwar recht unterschiedlich komponiert. Auf der Ebene ihrer ikonologischen Struktur (im Sinne der Wiederholung bestimmter Bildfolgen, Typen und ihres agitatorischen Potentials - unerheblich ob heroisierend oder diskriminierend) aber sind sie durchaus vergleichbar. In der Zusammenschau dieser beiden Ebenen, der Realismusfiktion einerseits und der verborgenen Symbolisation andererseits, kann von Fragmenten wie der Hakennase abstrahiert und ein weiterer semantischer Kontext (Hygiene, rassenanthropologische Messungen etc.) in Betracht gezogen werden.

Der Grund für die Strukturähnlichkeit von antiproletarischen und antijüdischen Bildern liegt in den Parallelen zwischen der Maschinisierung des proletarischen Alltags und der rationalen Steuerung des Volkskörpers: ein funktionierender Körper musste für die Fabriken geschaffen 
werden und sich in ihr Effizienzschema fügen, der ,neue Mensch“ sollte die Zukunft der Nation berechenbar machen. Die physische Bevölkerungsqualität wurde zu einer messbaren Größe, Abweichungen von diesen so genannten Binominalkurven, die durchschnittliche Prototypen generierten, wurden als negativa und Gefährdung interpretiert. Der Parallelität von Ökonomie und Biologie trug auch der italienische Anthropologe Alfredo Niceforo Rechnung, der sich eine Anthropologie des Sozialen zur Aufgabe gemacht hatte:

„Die ökonomische Produktion des Menschen kann also betrachtet werden als eine Funktion des Organismus, als ein Merkzeichen, das, wie alle anderen eine ruhende und absteigende Phase hat" (Niceforo 1910: 151).

Diese identifizierenden „Merkzeichen“ im Rahmen einer Wissenschaftskritik anzugreifen, verlangt zunächst eine Inventarisierung und Aneignung des Bildreservoirs in Form eines ikonografischen und darauf aufbauend eines ikonologischen Vergleichs. Dies bedeutet, sich einen Überblick darüber zu verschaffen, welche Zeichenelemente eingesetzt wurden, wie diese innerhalb einer Teilkultur wie z.B. der sozialdemokratischen Facharbeiterschaft (Lehnert 1989: 89-92) kontextualisiert waren und wie ihr darüber hinausgehendes Deutungspotential, der Überschuss (supplément im Sinne Derridas 1997: 114-139), beschaffen ist. Im Folgenden sollen die medikalisierten Bilder anhand dreier Muster der Körperdarstellung eingehender betrachtet werden: Industrialisierung, Pathologisierung und Dressur. Die Bilder werden dabei in der Analyse mit ihrem wissenschaftlichen bzw. sozio-kulturellen Entstehungskontext verknüpft.

\section{Der industrialisierte Körper}

Normalität (Link 1999: 15-26) und Rationalität sind als Charakteristika und Ideale der bürgerlichen Gesellschaft zu sehen. Die Nivellierung des arbeitenden Menschen auf einen reinen Leistungsfaktor führt in der Lesart Jürgen Links zu der Aussage, dass der Normalismus ein ,Epiphänomen“ des Kapitalismus sei, was angesichts der prototypischen Konzeption des Arbeiters im Bild von sozialistischer Seite bestärkt wird (ebd.: 235f.). Die Visualisierung des Proletariers aus christlichsozialer Sicht im Kikeriki hingegen weist deutliche Parallelen zu der des Juden auf, da beide als Fremdkörper konzipiert sind.

Der Mythos und das visuelle Stereotyp des gestählten, vitalen Arbeiters ist als Entfremdung der SPD-Parteispitze vom Arbeiteralltag und als 
realitätsbereinigte Kunstfigur, die das Selbstbild inszeniert, zu werten. Ein entscheidender Unterschied zwischen dem Wahren Jacob und Kikeriki besteht darin, dass im erstgenannten Medium eine Idealisierung und Heroisierung des Arbeiters, der autonom scheint, stattfindet, im letzteren dagegen die degenerativen Erscheinungen harter körperlicher Arbeit, die als ein Effekt von Ausbeutung durch Bolschewiken und Juden gehandelt wird, nachgezeichnet werden. Zum einen wirkt also die simulierte körperlich-seelische Gesundheit und Kraft, zum anderen die pathologisierte Existenz identifizierend. Die einem einfachen Grundmuster folgende Bildstruktur sorgt für die simultane Rezeption und das Ad-hocVerstehen antijüdischer und -proletarischer Bilder:

„Nur durch diese semantische Verknappung, durch die Stabilisierung von wenigen Deutungsvarianten ist gewährleistet, dass Symbole als zuverlässige Medien des Interdiskurses fungieren können. So gewinnen sie Wiedererkennungswert und unmittelbare Evidenz" (Dörner 1996: 45f.).

Für die Arbeiterkultur in der Weimarer Republik spielte die utopische Konzeption des Neuen Menschen eine gewichtige Rolle, dessen Zielvorgabe einer emanzipierten egalitären Gesellschaft, im Gegensatz zur amorphen kommunistischen Masse, ihm normative Macht verleihen sollte. Insbesondere das Verhältnis von Mensch und Maschine radikalisierte sich zu Beginn des 20. Jahrhunderts (vgl. den Beitrag von Michael Mackenzie in diesem Band), nicht zuletzt durch den Ersten Weltkrieg, in dem der Krieger zu einem entindividualisierten und seelenlosen Bediener von Waffen wurde (vgl. Theweleit: 158-162). In der bildenden Kunst ist diese Abrichtung des Menschen auf Reproduzierbarkeit (Kampfmaschine) und Funktionalität (Mechanisierung) in Form abstrakter, technischer Figuren in einem Gemälde von George Grosz mit dem Titel Der Neue Mensch (1921), röhrenähnlichen Körperkonstrukten in Fernand Légers Le déjeuner (1921), normierten Arbeiterfiguren im Ö1gemälde Die Arbeitsmänner (1925) von Franz W. Seiwert oder skulpturalen Tanzautomaten (bzw. -puppen) in Oskar Schlemmers Triadischem Ballett reflektiert und künstlerisch umgesetzt worden (vgl. Thomä 1999).

Auch Der Wahre Jacob illustriert „Die Mechanisierung des Arbeiters" (Abb. 2): Die zwischen den Akteuren, zwei dicken Kapitalisten, die durch Zigarre, Gamaschen und Frack als solche erkennbar sind, befindliche Arbeiterfigur gleicht einer eisernen Statue, die an den Maschinenmenschen aus Fritz Langs Metropolis (1927) erinnert. Ihr Herz, als symbolischer Lebensquell, wird durch einen berechen- und steuerbaren Mechanismus ersetzt. Sie sticht hervor durch ihre Nacktheit, ihren sym- 


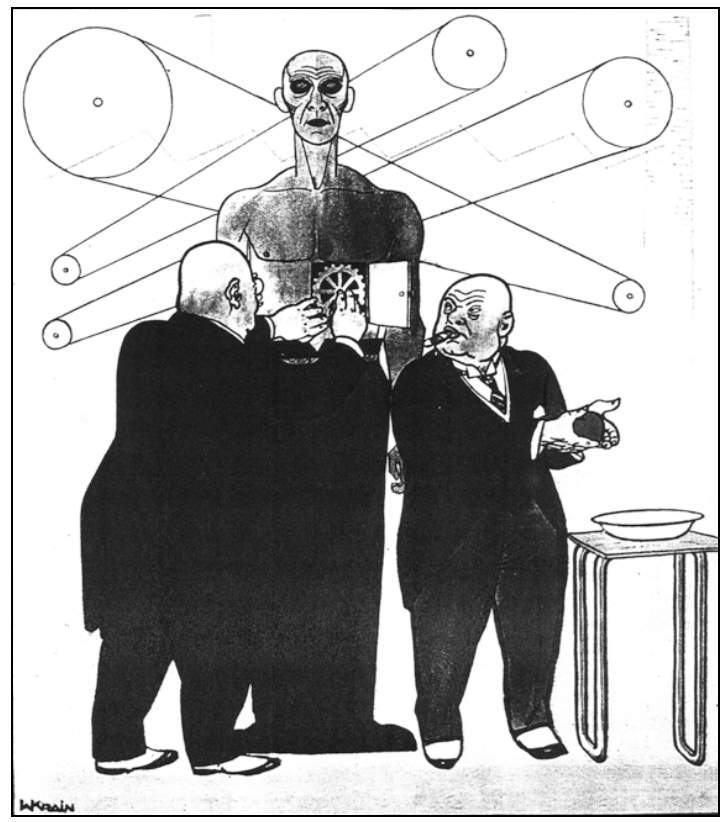

Abb. 2: „Die Mechanisierung des Arbeiters“. Der Wahre Jacob, 9. Juni 1928 (Beilage, Titelblatt).

metrischen Körper und vor allem den markanten unbehaarten Kopf mit den schwarzen Augenhöhlen, dem geöffneten Mund, den starken Wangenknochen und tiefen Stirnfalten. Die Unterzeile bezieht sich aber nicht auf die menschlichen Gefühle des Arbeiters, sondern auf eine reine Kosten-Nutzenrechnung: „Sie wollen den Menschen zur Maschine machen. - Wenn aber ein Mensch soviel kosten würde, wie eine Maschine, würden sie wahrscheinlich schnell wieder Abstand nehmen!“

In der Verklärung des „Malochers“ sind viele sozialistische, weniger christlichsoziale, Bilder der zwanziger Jahre mit einer Rohheit bzw. Ursprünglichkeit aufgeladen, die den zeitgenössischen Fordismus konterkarieren. Die zunehmende Spezialisierung und Professionalisierung auch des Handwerks, nicht nur der Bürokratie - wird in den beiden untersuchten Satirezeitschriften dagegen völlig ausgeblendet; Maschinen oder Steuerungspulte kommen in den Bildern nicht vor. Das Ideal der Symbiose von Heim und Arbeitsplatz und des familienverbundenen Arbeiters ist dem industriell vernutzten und ungesunden arbeitshygienischen Einflüssen ausgesetzten Fließbandarbeiter, der nicht kreativ Werte schöpft, sondern (re-)produziert und durch die ständige Routine ab- 
stumpft, entgegengesetzt. Der blue collar-Arbeiter ist nicht präsent, da kein schwarzmalerischer Realismus, sondern vielmehr ein positiver $\mathrm{Zu}-$ kunftsmythos erwünscht ist, ebenso wenig wie der assimilierte oder national-deutsch gesinnte Jude thematisiert wird, da dieser eigentlich , unsichtbar' ist. Es geht daher nicht um die Visualisierung von Normalität, sondern die bildliche Übersetzung einer Norm. In diesem Sinn betrachtet der zeitgenössische italienische Soziologe Roberto Michels die idealisierende Gestaltung des Arbeiters zum einen als die implizite Kritik am müßigen Wohlhabenden, zum anderen als das Sinnbild einer moralisch und physisch ,gesunden' Gesellschaft:

„Es gab eine Epoche in der Geschichte, in der die Weltverbesserer und Philosophen kein besseres Heilmittel für die Schäden der Zeit wussten, als dem entarteten, verweichlichten und krankhaft-kränklichen Reichen das Urbild des armen Mannes, wie es sich in ihren Köpfen malte, entgegenzuhalten. Das heißt den kräftigen und kerngesunden Armen, den Mann aus dem Volke mit den ,doppelten ' Muskeln, den prallen Backen und den kirschroten Lippen, zu dessen physischer Kraftfülle und sittlicher Tugend es zu gelangen galt“ (Michels 1910: 3).

Hier lässt sich eine Reminiszenz an den Rousseauschen homme naturel ablesen, dessen Glückseligkeit nur im vorindustriellen Naturzustand begründet war. Dem steht die pervertierte und automatisierte industrielle Gesellschaft gegenüber, deren Utopien am technischen Fortschritt orientiert sind. So drücken sich im Gegensatz zum ursprünglichen Idyll die Topoi von Luxus und Dekadenz in den im Kikeriki meist ,jüdisch“ markierten Kapitalisten - der Wahre Jacob zeichnet diese als ostelbische Junker - durch die Bildzeichen Diamanten, Taschenuhren und Ringe aus, als Armutszeichen hingegen gilt die zerlumpte und fleckige Kleidung, in die Ostjuden und Bolschewisten gleichermaßen gehüllt sind. Entsprechende Körperzeichen sind im ersten Fall Fettleibigkeit und eine antijüdische Physiognomie, im zweiten Magerkeit sowie eine faltige, pockige Haut, die auf Syphilis und Alkoholismus hinweisen sollte. Besonders die venerischen Krankheiten waren ein Vermächtnis des Krieges, standen aber auch für langfristige psychische Folgen für den „Volkskörper“, da Syphilis zur ,Hirnerweichung', im Wissenschaftsjargon dementia praecox, zu führen drohte. 


\section{Der pathologisierte Körper}

Die Bebilderung des Jüdischen oder des Proletarischen als Zeichen einer ,kranken Kultur' erscheint als eine experimentelle Anordnung, in der kein lebensweltliches Objekt (nach empirischen Studien und klassifizierenden Statistiken) vonnöten ist, um die Aussageabsicht des Künstlers/Wissenschaftlers zu prüfen bzw. zu widerlegen. Vielmehr wird im künstlichen Raum des Zeichenlabors ein virtueller Typus erzeugt, dem ideologische Strukturen im Bildaufbau zugrunde liegen. Ziel sowohl der Visualisierungen des Juden als auch des Arbeiters im Wahren Jacob und Kikeriki war es, ein Identifikations- bzw. Feindobjekt für die parteiliche Agitation zu schaffen, wobei in beiden Fällen der Authentizitätsanspruch eindeutig in den Hintergrund trat.

In den dichotomischen Konstruktionen des Kikeriki verschmelzen Proletarier und Juden, um sie mit Christen und Deutschen, die ebenfalls symbiotisch angelegt sind, kontrastieren zu können. In einem Bild des Kikeriki (Abb. 3), das auf ein Wahlplakat und Flugblatt der CSP (OENB 16315123, 1923/Kt. 3/2 und OENB 16307654, 1927/Kt. 7) anspielt, sitzt zur Rechten des anthropomorphen Kikeriki-Hahns friedlich und in häusliche Arbeit vertieft die ,heilige Familie', geschützt durch ein Kruzifix.

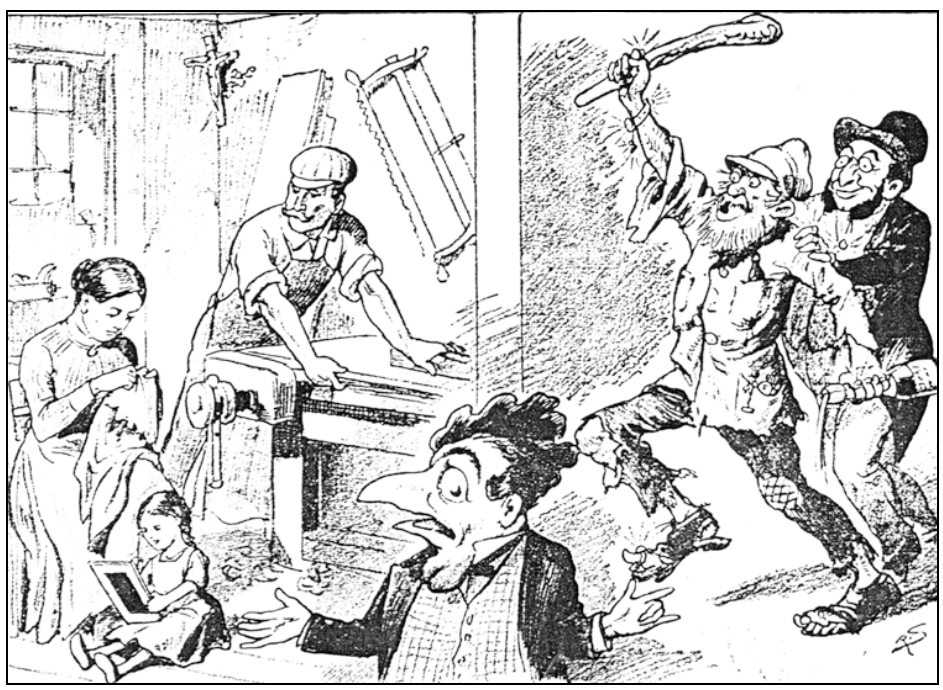

Abb. 3: Kikeriki, 26. Sept. 1920 (Titelblatt). 
Zur Linken tobt die ,unheilige Liaison` aus Bolschewist und ,jüdischem ‘ Kapitalist, animiert durch Champagner. Die Akteure sind jüdischbolschewistisch konnotiert, die Erleidenden, in ihr Schicksal Ergebenen christlich, was den Kontrast von Ruhe und Raserei noch verstärkt und mit der häufig applizierten Opfermetaphorik des Kikeriki korrespondiert. Gewalt und Dekadenz konterkarieren und bedrohen hier Andächtigkeit und Fleiß. Man könnte diese visuellen Kombinationen als Feindbildcluster, die das singuläre Stereotyp gezielt aufladen, bezeichnen.

So trifft sich in den 1920er Jahren der bildliche antijüdische Phänotyp mit der medizinischen Diagnostik des degenerierten Arbeiter(körper)s, woran - wie eingangs erwähnt - ersichtlich ist, wie sich die Diskurse der Differenz verzahnen. Das äußere Erscheinungsbild des Arbeiters - in der Empirie - ist dem zeitgenössischen Anthropologen Alfredo Niceforo zufolge im Wesentlichen durch die folgenden Charakteristika gekennzeichnet: a) eine geringere Körpergröße im Vergleich zu den wohlhabenden Klassen, b) den „Ausdruck allgemeiner Erschlaffung und physischer Dekadenz" (Niceforo 1910: 135), vor allem was die Physiognomie betrifft, c) eine Übereinstimmung des Gesichtswinkels en profil und en face mit dem „Neger- und Mongolentypus“ (ebd.: 139f.), was Niceforo auf Überanstrengung, schlechte Ernährung und Vergiftungen des Foetus im Mutterleib zurückführt, d) ein geringeres Vorkommen von Kahlköpfigkeit und Ergrauen, dafür aber vorzeitige Hautalterung, d.h. Faltenbildung, e) Unterernährung, womit Niceforo dem Vorurteil von den generell muskelhypertrophen Arbeitern widerspricht, und schließlich f) ein ,tuberkulöser Habitus, Engbrüstigkeit, Magerkeit und blasse Farbe" (ebd.: 144f.). Der letzte Punkt wird noch eine Rolle im militärischen Kontext spielen.

Indem Niceforo zwischen dem degenerierten „Neger- und Mongolentypus“ und dem europäischen Typus, der zur „weißen Rasse“ gehöre, differenziert, stigmatisiert er die Arbeiter wegen ihrer visuellen Kohärenz mit einem als minderwertig betrachteten „Rassetypus“. Er verweist auf seine Inspiration durch die fotografischen Studien Guillaume Benjamin Duchennes an der Pariser Salpêtrière, einer psychiatrischen Heilanstalt, an der Jean-Martin Charcot, der Lehrer Siegmund Freuds (vgl. Didi-Huberman 1997), durch elektrische Impulse Gesichtsmimiken, Gesichtsspasmen und ähnliches hervorrief und dokumentierte. Der Gesichtsausdruck sollte Aufschluss über geistige Störungen und Krankheiten geben. Nicht zufällig strahlen auch die stereotypen Gesichter von Juden und Proletariern in den besprochenen Populärmedien oft Demenz und „Äffisches“ aus; das Vorurteil mangelnder Triebkontrolle und geistiger Unberechenbarkeit verklammerte somit rassische und soziale Stigmata. 
Niceforo fasst als physiognomische und kraniometrische „Anomalieen“, wie sie häufig bei den „nichtbesitzenden Klassen“ vorkämen, (Niceforo 1910: 136) etwa Plagiocephalie, die zurücktretende Stirn, Prognatismus, Asymmetrie des Gesichtes, übermäßig große Kiefer, anormale Ohren (Henkelohren oder „,knollenartige“, unsymmetrische Ohren), stark entwickelte Augenbrauen und hervorspringende Wangenknochen. Henkelohren fanden häufigen Gebrauch in antijüdischen Karikaturen, um kriminelle Leidenschaften zu suggerieren, und gehen auf Typologien zurück, die bereits der italienische Kriminalanthropologe Cesare Lombroso entwickelt hatte (Lombroso 1887: 384-400). Um die weiter oben angestellten Überlegungen zum bürgerlichen Normalisierungsprozess aufzugreifen: Ins Gewicht fallen die optischen Defekte, die sich nicht an Arbeitern derselben Branche orientieren, sondern am (Büro-)Angestellten, dem Normalbürger, der in seinem white collar job keinen giftigen Dämpfen, keinem maschinellen Lärm oder dem Schichtbetrieb ausgesetzt ist. Die durch Selbstdisziplin und Gesundheitsbewusstsein charakterisierte Angestelltenschaft des frühen 20. Jahrhunderts bildet die unsichtbare Norm einer fiktiven Nationalmoral hinter den Bildern (Föllmer 2001: 41-67).

Dementsprechend hielt auch der Düsseldorfer Arzt und Privatdozent Voss das Milieu in Bezug auf nervöse Leiden für bedeutsamer denn genetische Dispositionen (Voss 1977: 401). Er nimmt somit den Diskurs auf, der den Naturmenschen mit dem Kulturmenschen konfrontiert, die Stadt als Brutstätte des Alkoholismus, der Syphilis und Tuberkulose verortet und Migranten, d.h. insbesondere Juden und Wanderarbeiter, als anfälliger für Psychosen erklärt (vgl. Weindling 2000: 6). Entscheidend für die Interpretation der Körperkonstrukte von Juden und Proletariern ist, dass hier von einer gemeinsamen Mentalität ausgegangen wurde. Im Falle des Kikeriki ist diese, wie gezeigt, auch visualisiert.

\section{Der dressierte Körper}

Betrachtet man die Berichte von Wehrtauglichkeitsuntersuchungen der zwanziger Jahre, so trifft sich auch hier eine diskriminierende Semantik des neurasthenischen und bereits erwähnten „engbrüstigen“ Juden mit der des degenerierten Arbeiters - ähnlich wie weiter oben bei Niceforo diagnostiziert. Die Skepsis in die Wehrtauglichkeit und damit das Nationalbewusstsein und die „Vaterlandstreue“ der (sub)proletarischen Bevölkerung, wozu in Teilen auch Juden - insbesondere die aus Osteuropa emigrierten - zählten, drückte sich in der sozialdarwinistischen pessimistischen Auffassung aus, der Krieg vernichte die Starken und erhalte 
die Schwachen. Das Moment des Krieges als eines biopolitischen Katalysators, der den Volkskörper reinigen könne, wird hier nicht genutzt, wie der Militärarzt Max Nonne in einem Handbuch für Ärzte bemängelt:

„Die besten werden geopfert, die körperlich und geistig Minderwertigen, Nutzlosen und Schädlinge werden sorgfältig konserviert, anstatt daß bei dieser günstigen Gelegenheit eine gründliche Katharsis stattgefunden hätte, die zudem durch den Glorienschein des Heldentodes die an der Volkskraft zehrenden Parasiten verklärt hätte“ (Nonne 1922: 112).

Die Entstehung und Verbreitung der Degenerationskonzepte und am Anfang des 20. Jahrhunderts auch der Sozial-/Rassenhygiene steht mit dem tiefgreifenden sozialstrukturellen Wandel der Industriegesellschaft im Zusammenhang. Zwei Faktoren beeinflussen dabei die pathologisierte Darstellung des jüdischen bzw. proletarischen Körpers wesentlich. Erstens trug die mangelhafte Wohnsituation zur Verbreitung von Krankheiten bei, wie etwa das Fehlen einer Heizung, feuchte und dunkle Räume, die schlecht gelüftet wurden und verantwortlich für Rachitis und (infolge des Vitaminmangels) für Skorbut waren, der Abort auf dem Treppenpodest oder im Hof. Die Popularisierung des rachitischen klinischen Bildes kann an den O-/X-Beinen, von denen viele Moulagen aus den 1920er Jahren im Dresdner Hygienemuseum archiviert sind, der Skorbut an der blassen bis gelblichen Gesichtsfarbe in den eigentlich satirischen und nicht primär (oder besser: explizit) wissenschaftlichen Bildern nachgewiesen werden.

Zweitens kamen zu der mangelhaften Ernährung schlechte Arbeitsbedingungen hinzu, was die Militärtauglichkeit v.a. in den Arbeiterbezirken reduzierte. Der Rassenhygieniker Alfred Ploetz merkte diese Ungleichheit und idealisierte Wahrnehmung des Arbeiterlebens an, die sich in der überproportionalen Mortalität der Arbeiter niederschlug:

„Man hört so oft von der Gesundheit reden, die dem Armen als Lohn für seine einfache Lebensweise zu theil wird, demgegenüber der Kränklichkeit des Wohlhabenden in Folge üppiger Lebensweise, und tritt auch wohl manchmal mit dieser Behauptung allzu unzufriedenen Elementen entgegen. Allein die Statistik lehrt doch etwas ganz Anderes“(Ploetz 1895: 157; vgl. Fischer 1909: 471-487).

Meist aufgrund statistischer Daten, die man von der Medizinalstatistik der Gesundheitsämter oder einzelnen „Irrenhäusern“ auf der Grundlage nosologischer Bestimmungen seitens einzelner Ärzte bezog, formierte sich ein bestimmtes Krankheitsbild, das wiederum auf spezifische Berufsgruppen und konstruierte „Rassen“ (vgl. Hanke 2000: 181) abhob. 
So differenzierten einzelne Studien die Berufsgruppen nach ihrem Hirngewicht, wobei einige feststellten, dass das der Arbeiter deutlich unter dem von Beamten läge oder Geistliche weniger häufig zur Geisteskrankheit neigten als „Metall- und Eisenarbeiter“ (Voss 1977: 401). Der Arzt Elias Auerbach nahm die Militärtauglichkeit als Gradmesser für die „körperliche Beschaffenheit der Bevölkerung“, wobei die jüdischen Organisationen sich der „,körperlichen Hebung der Juden“ - aufgrund der leicht überproportionalen Untauglichkeit - widmen sollten (vgl. Auerbach 1908: 187-189).

„Und nun bedenke man die soziale Schichtung der Juden in Deutschland! Keine Ackerbauer, keine Schiffer, keine Steinhauer, Kutscher, selbst kaum Schlosser, Böttcher, Zimmerleute - dafür massenhaft Buchhalter, Händler, Schneider, Studierte. Die Entwicklung, die dahin geführt hat, datiert nicht von heute, die ganze Zeit des Ghettos war die Vorschule dazu. Da haben die Juden das tiefe Atmen in der freien Luft verlernt“ (Auerbach 1908: 189).

Auerbach gelangt zu der Forderung, dass durch sportliche Ertüchtigung und Hinwendung zu ,gesunden Berufen“ der jüdische „Stamm“ gestärkt werden müsse. Die Perspektive des Landerwerbs versprach Heilung des ,jüdischen Volkskörpers“, verbunden mit der Arbeit der Juden auf der „eigenen Scholle“, was sich in etlichen Landwirtschaftsschulen, die auf die Alijah, die Auswanderung nach Eretz Israel, vorbereiten sollten, ausdrückte. Der Rabbiner Leo Baeck forderte in diesem Sinne eine Rückkehr zur mentalen und körperlichen Gesundheit durch den „Weg zum Boden“, der „Heilung und Befreiung“ garantiere und die Oberflächlichkeit und Missachtung vor Wachsendem und Gewordenem ablege (Baeck 1931: 68). Interessanterweise ist diese ,Bodenprosa' auch im sozialdemokratischen Diskurs zu finden, die den Arbeiter als Menschen wieder in ein quasi-natürliches Umfeld re-integrieren möchte, wie man an den Programmen der Volkshochschulen und Wanderkurse ablesen kann. Beide Parteien zielten daher auf eine lebensweltliche Verwurzelung der jeweiligen Klientel und zeigten umgekehrt die tiefe Entfremdung von der nach dem Ersten Weltkrieg radikalisierten deutschen bzw. österreichischen Nation.

Eine schwächliche und effeminierte Konstitution, die im Widerspruch zum vitalen und virilen Soldatenkörper steht, erhält als Bildmittel politische Relevanz und war dabei vor allem von Wehrtauglichkeitsnormen bestimmt, die zum Eintrittsbillet in die deutsch-nationale Gesellschaft wurden (Hödl 1997: 168-177). Zum schmächtigen Oberkörper von Juden und Proletariern kam noch die unterstellte „Plattfüßigkeit“, was das Negativurteil „marschuntauglich“ nach sich zog (vgl. Muskat 
1907: 318-331; ders. 1926: 682; ders. 1928: 234). Auf dem Kongress der deutschen Gesellschaft für Chirurgie (1928) befanden aber einige Orthopäden auf der Grundlage von Wehrtauglichkeitsstatistiken und anhand von Praxisberichten übereinstimmend, dass der Plattfuß keine „Rasseneigentümlichkeit“, sondern eine „Volkskrankheit“ sei. Durch eine Volkserziehung, die das Zusammenwirken von Elternhaus, Schule und Arzt vorsah, sollte eine Vermeidung des Plattfußes - wie er besonders häufig bei bestimmten Berufsgruppen (z.B. Kellnern, Bäckern, Schmieden sowie Lasten tragenden Arbeitern v.a. in der Landwirtschaft) auftrat - vermieden werden, um die ,jüdische Bevölkerung von dem Odium des nur ihr zukommenden Plattfußes befreien“ (Muskat 1928: 234).

\section{Die Körpertypologien - ein Resümée}

Die christlichsoziale Zeitschrift Kikeriki wendet degenerative Attribute sowohl auf den Juden auch auf den Arbeiter an und zeichnet letzteren als kranken Bolschewisten. Ebenso wurden hier Sozialisten (bzw. Bolschewisten oder auch Kommunisten) wie Juden als heimatlos und supranational (im Anklang an den Ahasver-Mythos) charakterisiert, weshalb viele Bilder des Kikeriki ein unsichtbares Band zwischen angeblich destruktiven Juden und Bolschewisten - im Kontrast zum produktiven ,deutschen Arbeiter“- weben. Die „Mentalität des Kapitalismus“, nach Werner Sombart, manifestierte sich in der Entwurzelung sowohl des Proletariers als auch des Juden sowie in der „Entzweiung“ des Denkens.

„,Der Proletarier hat kein Vaterland‘. Heimatlos, rastlos wird er auf der Erde umhergetrieben. [...] Seine Heimat ist die Welt. Er ist ein Allerweltsmensch. [...] Zerstört durch den eisernen Fuß des Kapitalismus sind alle jene Gemeinschaften, die den Menschen der früheren Zeit, vor allem den Durchschnittsmenschen der großen Masse, zwar banden, aber auch hielten, stützten, wirtschaftlich und moralisch“ (Sombart 1906: 12f.).

Im sozialdemokratischen Wahren Jacob dagegen sind die negativen, degenerativen Attribute der medizinischen Diagnostik einseitig auf den Juden, der als Prototyp des homo urbanus und des mittleren Bürgers galt, bezogen, während man den Arbeiter seiner aktuellen Gefährdungen und Berufskrankheiten entkleidete und ihn paradoxerweise als gesunde Norm visualisierte. Die Kollektivsymbole „Arbeiter“ und „Juden“ in dieser Zeitschrift sollen resümierend gegenüber gestellt werden: Der prototypisch konstruierte Arbeiter ist meist mit den folgenden visuellen 
Attributen - sachlicher und körperlicher Art - ausgestattet: a) kräftige, muskulöse Statur, b) geballte Fäuste, in denen er manchmal auch eine Fahne oder Werkzeug hält, c) eine breitbeinige Pose, meist in der Frontalansicht und d) ein kantiges Gesicht, in dem das breite Kinn, die leicht hohlen Wangen, die tiefen Augenhöhlen, zusammengezogene Brauen und fest geschlossene Lippen mit leicht herabgezogenen Mundwinkeln markant hervorstechen. Im Gegensatz dazu ist der (anti-)jüdische Prototyp eine Mischung aus Attributen „des“ Ostjuden, der sich stets durch ein Zeichenrepertoire aus Kippa, Tefilin, Peies, einem langen Bart und überzeichneter Physiognomie konstituiert, und rassenanthropologischen ,Befunden': a) gekrümmte Statur, b) überdimensionierte, gestikulierende Hände, c) O-/X-Beine, d) ein meist deformierter Kopf mit großem Gesichtsschädel, Hakennase, Prognatie (Schnauzenförmigkeit), kleinen und engstehenden Augen, schweren Augenlidern und wulstigen Lippen.

Der Versuch, die Nachkriegsgesellschaften entweder sozial oder rassisch zu stratifizieren, entsprang einem Bedürfnis nach Identifizierung und Abgrenzung vom politischen Gegner, in den untersuchten Zeitschriften in Bezug auf die SPD und CSP. Die visuellen Bilder von ,jüdischen' und ,proletarischen“ Körpern arbeiten gezielt mit dem Verfahren und der Lust an der optischen Diagnose und existentiellen Ängsten, die von der Übervölkerung durch Unterschichten oder so genannte ,Degenerierte' bis hin zur körperlichen oder moralischen ,Infizierung' reicht. Letztlich gibt es aber keine gesonderte Visualisierung des ,Abjekts', vielmehr funktioniert dies nach denselben Regeln und Techniken wie die normativen Elemente einer Kultur. Anders gesagt: In der Repräsentation von Juden und Proletariern handelt es sich zwar um äußerlich unterschiedliche Phänomene, die Visualisierungsstrategie jedoch, nämlich den politischen Gegner zu pathologisieren bzw. eine idealisierte Leitfigur zu schaffen, und die Wirkungsstruktur, die auf den stereotypen Symbolen als Popularisierungs- und Politisierungsmittel aufbaut, sind gleich angelegt.

\section{Literaturverzeichnis}

Auerbach, Erich (1908): „Über die Militärtauglichkeit der Juden“. Jüdische Turnzeitung 10/11, S. 187-189.

Baeck, Leo (1931): „Mensch und Boden. Gedanken zur Soziologie des Großstadtjuden“. Der Schild 9 (14. Mai), S. 68.

Becker, Frank/Gerhardt, Ute/Link, Jürgen (1997): „Moderne Kollektivsymbolik. Ein diskurstheoretischer Forschungsbericht mit Auswahl- 
bibliographie. Teil II“. Internationales Archiv für Sozialgeschichte der deutschen Literatur 22, S. 70-154.

Berliner Klinische Wochenschrift (1898).

Derrida, Jacques (1997): „Die Struktur, das Zeichen und das Spiel“. In:

Peter Engelmann (Hg.), Postmoderne und Dekonstruktion. Texte französischer Philosophen der Gegenwart, Stuttgart: Reclam, S. 114139.

Didi-Huberman, George (1997): Erfindung der Hysterie. Die photographische Klinik von Jean-Martin Charcot, München: Fink.

Dörner, Andreas (1996): Politischer Mythos und symbolische Politik. Der Hermannsmythos: Zur Entstehung des Nationalbewußtseins der Deutschen, Reinbek: rororo.

Drews, Arne/Link, Jürgen/Gerhardt, Ute (1985): „Moderne kollektive Symbolik. Eine diskurstheoretisch orientierte Einführung mit Auswahlbibliographie“. Internationales Archiv für Sozialgeschichte der deutschen Literatur (Sonderheft I), S. 256-375.

Fischer, Alfons (1909): „Rekrutenstatistik und Volksgesundheit“. Jahrbücher für Nationalökonomie und Statistik 93 (III. Folge, 38. Band), S. 471-487.

Föllmer, Moritz (2001): „Der ,kranke Volkskörper‘. Industrielle, hohe Beamte und der Diskurs der nationalen Regeneration in der Weimarer Republik“. Geschichte und Gesellschaft 27, S. 41-67.

Friedrich, Ernst (2004): Krieg dem Kriege, Reprint [Original 1924], München: DVA.

Gilman, Sander L. (2000): „Das Gesicht wahren. Zur ästhetischen Chirurgie“. In: Claudia Schmölders/ders. (Hg.), Gesichter der Weimarer Republik. Eine physiognomische Kulturgeschichte, Köln: DuMont, S. 96-112.

Hanke, Christine (2000): ,Zwischen Evidenz und Leere. Zur Konstitution von ,Rasse“ im physisch-anthropologischen Diskurs um 1900“. In: Hannelore Bublitz/Christine Hanke/Andrea Seier (Hg.), Der Gesellschaftskörper. Zur Neuordnung von Kultur und Geschlecht um 1900, Frankfurt a.M./NewYork: Campus, S. 179-235.

Hödl, Klaus (1997): Die Pathologisierung des jüdischen Körpers. Antisemitismus, Geschlecht und Medizin im Fin de Siècle, Wien: Picus.

Kikeriki (1919).

Lehnert, Detlef (1989): „,Staatspartei der Republik‘ oder ,revolutionäre Reformisten“?““. In: Ders./Klaus Megerle (Hg.), Politische Identität und nationale Gedenktage: Zur politischen Kultur in der Weimarer Republik, Opladen: Westdeutscher Verlag, S. 89-92. 
Link, Jürgen (1999): Versuch über den Normalismus: Wie Normalität produziert wird, 2. akt. u. erw. Aufl., Opladen: Westdeutscher Verlag.

Lombroso, Cesare (1887): Der Verbrecher (Homo delinquens) in anthropologischer, ärztlicher und juristischer Beziehung, in deutscher Bearbeitung von M.O. Fraenkel, 1. Band, Hamburg: Verlagsanstalt.

Michels, Robert (1910): „Das Proletariat in der Wissenschaft und die ökonomisch-anthroplogische Synthese (Einführung)“. In: Alfredo Niceforo, Anthropologie der nichtbesitzenden Klassen. Studien und Untersuchungen, Leipzig/Amsterdam: Maas \& Van Suchtelen.

Muskat, Gustav (1907): „Die Verhütung des Plattfußes mit besonderer Berücksichtigung der Wehrfähigkeit“. Beilage zur Hygienischen Rundschau 21, S. 318-331.

Muskat, Gustav (1926): „Ist der Plattfuß eine ,Rasseneigentümlichkeit"?" C.V.-Zeitung 5, S. 682.

Muskat, Gustav (1928): „Der Plattfuß als Volkskrankheit“. Der Schild 30 (28. Juli), S. 234.

Niceforo, Alfredo (1910): Anthropologie der nichtbesitzenden Klassen. Studien und Untersuchungen, Leipzig/Amsterdam: Maas \& Van Suchtelen.

Nonne, Max (1922): „Therapeutische Erfahrungen an den Kriegsneurosen in den Jahren 1914 bis 1918“. In: Karl Bonhoeffer (Hg.), Handbuch der Ärztlichen Erfahrungen im Weltkriege 1914/1918, Band IV, Leipzig: Barth.

Ploetz, Alfred (1895): Die Tüchtigkeit unserer Rasse und der Schutz der Schwachen, Berlin: Fischer.

Schäfer, Julia (2004): „Ein Jude ist ein Jude ist ein Jude - Das Judenbild zwischen Mimesis und Inszenierung“. In: Katja Bär/Kai Berkes/Stefanie Eichler u.a. (Hg.), Text und Wahrheit, Frankfurt a.M. u.a.: Peter Lang, S. 209-224.

Schäfer, Julia (2005): Vermessen - gezeichnet - verlacht. Judenbilder in populären Zeitschriften 1918-1933, Frankfurt a.M./New York: Campus.

Schlemiel. Witzblatt für jüdischen Humor (1919).

Sombart, Werner (1906): Das Proletariat. Bilder und Studien, Frankfurt a.M.: Rütten und Loening.

Theweleit, Klaus (1995): Männerphantasien, Band 2: Zur Psychoanalyse des weißen Terrors, München: dtv.

Thomä, Dieter (1999): „Freiheit und Glück“. In: Nicola Lepp/Martin Roth/Klaus Vogel (Hg.), Der neue Mensch. Obsessionen des 20. Jahrhunderts, Stuttgart: Cantz, S. 91-101. 
Voss, G. (1977): „Der Einfluß der sozialen Lage auf Nerven- und Geisteskrankheiten, Selbstmord und Verbrechen“ [Original 1913]. In: Max Mosse/Gustav Tugendreich (Hg.), Krankheit und soziale Lage, Göttingen: Jürgen Cromm Selbstverlag, S. 400-472.

Wahre Jacob, Der (1928).

Weindling, Paul Julian (2000): Epidemics and Genocide in Eastern Europe, 1890-1945, Oxford: Oxford University Press. 